\title{
First AGILE catalog of high-confidence gamma-ray sources
}

C. Pittori ${ }^{1}$, F. Verrecchia ${ }^{1}$, A. W. Chen ${ }^{2,3}$, A. Bulgarelli ${ }^{4}$, A. Pellizzoni ${ }^{5}$, A. Giuliani ${ }^{2,3}$, S. Vercellone ${ }^{6}$, F. Longo ${ }^{7,8}$, M. Tavani ${ }^{9}, 10,11,3$, P. Giommi ${ }^{1,12}$, G. Barbiellini ${ }^{7,8,3}$, M. Trifoglio ${ }^{4}$, F. Gianotti ${ }^{4}$, A. Argan ${ }^{9}$, A. Antonelli ${ }^{13}$, F. Boffelli ${ }^{14}$, P. Caraveo ${ }^{2}$, P. W. Cattaneo ${ }^{14}$, V. Cocco ${ }^{10}$, S. Colafrancesco ${ }^{1,12}$, T. Contessi ${ }^{2}$, E. Costa ${ }^{9}$, S. Cutini ${ }^{1}$, F. D’Ammando ${ }^{9,10}$, E. Del Monte ${ }^{9}$, G. De Paris ${ }^{9}$, G. Di Cocco ${ }^{4}$, G. Di Persio 9 , I. Donnarumma ${ }^{9}$, Y. Evangelista ${ }^{9}$, G. Fanari ${ }^{1}$, M. Feroci ${ }^{9}$,

A. Ferrari ${ }^{3,15}$, M. Fiorini ${ }^{2}$, F. Fornari ${ }^{2}$, F. Fuschino ${ }^{4}$, T. Froysland ${ }^{3,11}$, M. Frutti ${ }^{9}$, M. Galli ${ }^{16}$, D. Gasparrini ${ }^{1}$, C. Labanti ${ }^{4}$, I. Lapshov ${ }^{9,17}$, F. Lazzarotto ${ }^{9}$, F. Liello9 ${ }^{9}$, P. Lipari ${ }^{18,19}$, E. Mattaini ${ }^{2}$, M. Marisaldi ${ }^{4}$,

M. Mastropietro ${ }^{9,21}$, A. Mauri ${ }^{4}$, F. Mauri ${ }^{14}$, S. Mereghetti $^{2}$, E. Morelli ${ }^{4}$, E. Moretti ${ }^{7,8}$, A. Morselli ${ }^{11}$,

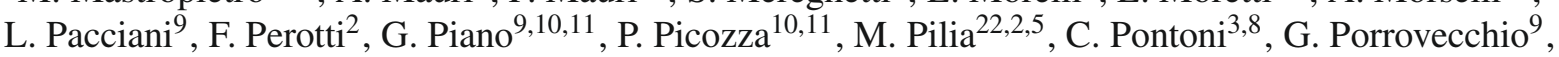
B. Preger ${ }^{1}$, M. Prest ${ }^{8,22}$, R. Primavera ${ }^{1}$, G. Pucella ${ }^{9}$, M. Rapisarda ${ }^{20}$, A. Rappoldi ${ }^{14}$, E. Rossi ${ }^{4}$, A. Rubini ${ }^{9}$, S. Sabatini ${ }^{10}$, P. Santolamazza ${ }^{1}$, E. Scalise ${ }^{9}$, P. Soffitta ${ }^{9}$, S. Stellato ${ }^{1}$, E. Striani ${ }^{10}$, F. Tamburelli ${ }^{1}$, A. Traci ${ }^{4}$, A. Trois $^{9}$, E. Vallazza ${ }^{8}$, V. Vittorini ${ }^{9,3}$, A. Zambra ${ }^{2,3}$, D. Zanello ${ }^{18,19}$, and L. Salotti ${ }^{12}$

1 ASI Science Data Center, ESRIN, 00044 Frascati (RM), Italy e-mail: pittori@asdc.asi.it

2 INAF-IASF Milano, via E. Bassini 15, 20133 Milano, Italy

3 Consorzio Interuniversitario Fisica Spaziale (CIFS), villa Gualino - v.le Settimio Severo 63, 10133 Torino, Italy

4 INAF-IASF Bologna, via Gobetti 101, 40129 Bologna, Italy

5 Osservatorio Astronomico di Cagliari, loc. Poggio dei Pini, strada 54, 09012, Capoterra (CA), Italy

6 INAF-IASF Palermo, via Ugo La Malfa 153, 90146 Palermo, Italy

Dip. Fisica, Università di Trieste, via A. Valerio 2, 34127 Trieste, Italy

INFN Trieste, Padriciano 99, 34012 Trieste, Italy

INAF-IASF Roma, via del Fosso del Cavaliere 100, 00133 Roma, Italy

Dipartimento di Fisica, Università Tor Vergata, via della Ricerca Scientifica 1, 00133 Roma, Italy

1 INFN Roma Tor Vergata, via della Ricerca Scientifica 1, 00133 Roma, Italy

2 Agenzia Spaziale Italiana, viale Liegi 26, 00198 Roma, Italy

3 Osservatorio Astronomico di Roma, via di Frascati 33, 00040 Monte Porzio Catone, Italy

4 INFN Pavia, via Bassi 6, 27100 Pavia, Italy

15 Dipartimento di Fisica, Università di Torino, via P. Giuria 15, 10126 Torino, Italy

16 ENEA Bologna, via don Fiammelli 2, 40128 Bologna, Italy

17 IKI, Moscow, Russia

18 INFN Roma 1, p.le Aldo Moro 2, 00185 Roma, Italy

19 Dip. Fisica, Università La Sapienza, p.le Aldo Moro 2, 00185 Roma, Italy

20 ENEA Frascati, via Enrico Fermi 45, 00044 Frascati (RM), Italy

21 CNR, IMIP, Area Ricerca Montelibretti (RM), Italy

22 Dip. Fisica, Università dell'Insubria, via Valleggio 11, 22100 Como, Italy

Received 4 February 2009 / Accepted 3 August 2009

\section{ABSTRACT}

We present the first catalog of high-confidence $\gamma$-ray sources detected by the AGILE satellite during observations performed from July 9, 2007 to June 30, 2008. Cataloged sources were detected by merging all the available data over the entire time period. AGILE, launched in April 2007, is an ASI mission devoted to $\gamma$-ray observations in the $30 \mathrm{MeV}-50 \mathrm{GeV}$ energy range, with simultaneous X-ray imaging capability in the $18-60 \mathrm{keV}$ band. This catalog is based on Gamma-Ray Imaging Detector (GRID) data for energies greater than $100 \mathrm{MeV}$. For the first AGILE catalog, we adopted a conservative analysis, with a high-quality event filter optimized to select $\gamma$-ray events within the central zone of the instrument field of view (radius of $40^{\circ}$ ). This is a significance-limited $(4 \sigma)$ catalog, and it is not a complete flux-limited sample due to the non-uniform first-year AGILE sky coverage. The catalog includes 47 sources, 21 of which are associated with confirmed or candidate pulsars, 13 with blazars (7 FSRQ, 4 BL Lacs, 2 unknown type), 2 with HMXRBs, 2 with SNRs, 1 with a colliding-wind binary system, and 8 with unidentified sources.

Key words. gamma rays: observations - catalogs

\section{Introduction}

AGILE (Astrorivelatore Gamma ad Immagini LEggero) (Tavani et al. 2008, 2009a) is a mission of the Italian Space Agency (ASI) devoted to $\gamma$-ray astrophysics in the $30 \mathrm{MeV}-50 \mathrm{GeV}$ and
18-60 keV energy ranges. AGILE was successfully launched on April 23, 2007 in a $\sim 550 \mathrm{~km}$ equatorial orbit with low inclination angle, $\sim 2.5^{\circ}$. High-energy $\gamma$-ray astrophysics is entering a new challenging phase of discovery. During the 1970's and 1980's, the SAS-2 (Fichtel et al. 1975) and COS-B 


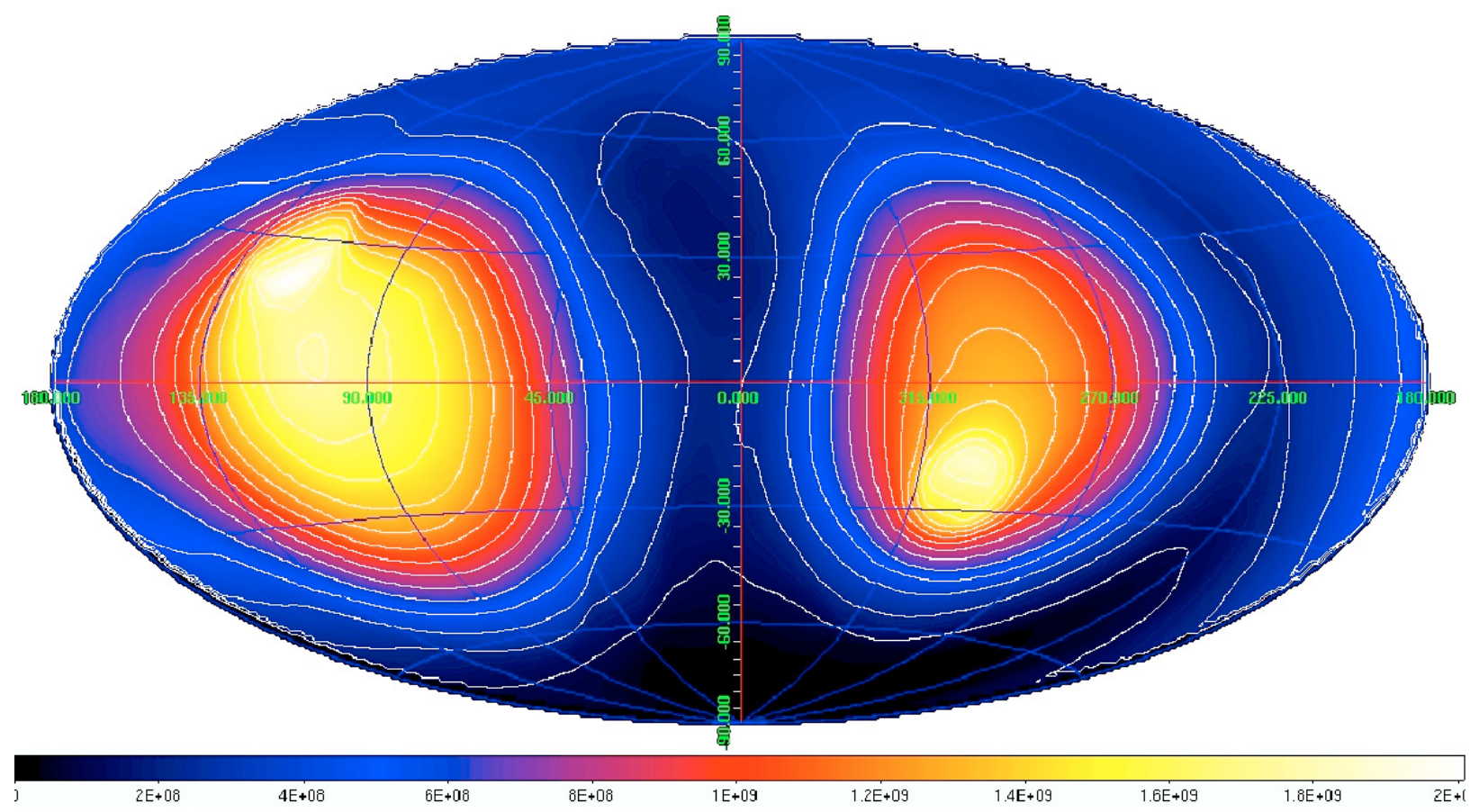

Fig. 1. Total AGILE-GRID exposure sky map in Aitoff projection and Galactic coordinates, for energies $>100 \mathrm{MeV}$ in units of $\mathrm{cm}^{2} \mathrm{~s}$, accumulated during the period July 9, 2007-June 30, 2008 (with the F4 event filter). The regions of deeper exposures (whiter in the color scale) are a consequence of the AGILE specific pointings at the Galactic plane, combined with the effect of Earth occultation.

(Bignami et al. 1975; Bennet et al. 1977) space missions discovered the very first cosmic $\gamma$-ray sources around $100 \mathrm{MeV}$, but our knowledge of high-energy cosmic $\gamma$-ray emission and phenomena up to now was mainly based on the remarkable results obtained by the EGRET instrument, onboard the Compton Gamma-Ray Observatory (CGRO) (Thompson et al. 1993). Nearly $300 \gamma$-ray sources above $100 \mathrm{MeV}$ were detected by EGRET (Hartman et al. 1999) during the period from April 22, 1991 to October 3, 1995; however, only a small fraction of them $(\sim 30 \%)$ have currently been identified. Many sources are variable or transient on short timescales, and our understanding of many high energy phenomena is still preliminary.

AGILE is the first $\gamma$-ray mission operating in space after almost ten years since the end of EGRET operations. AGILE was the only mission entirely dedicated to high-energy astrophysics above $30 \mathrm{MeV}$ during the period April 2007-June 2008. It is currently operating together with the Fermi Gamma-Ray Space Telescope (formerly GLAST), launched on June 11, 2008 (Michelson 2008; Atwood et al. 2009). The highly innovative AGILE instrument is the first of a new generation of high-energy space missions based on solid-state silicon technology, expected to substantially advance our knowledge in several research areas including the study of active galactic nuclei, gamma-ray bursts, pulsars, unidentified $\gamma$-ray sources, Galactic compact objects, supernova remnants, etc.

The AGILE Payload detector consists of the silicon tracker (ST) (Barbiellini et al. 2001; Prest et al. 2003), the X-ray detector SuperAGILE (Feroci et al. 2007), the CsI(Tl) Mini-Calorimeter (MCAL) (Labanti et al. 2006), and an anticoincidence system (ACS) (Perotti et al. 2006). The combination of ST, MCAL, and ACS forms the Gamma-Ray Imaging Detector (GRID). Accurate timing, positional and attitude information is provided by the precise positioning system and the two star sensors units. The silicon tracker, based on the process of photon conversion into electron-positron pairs, is the core of the AGILE-GRID. It consists of a total of 12 trays, the first 10 of which are capable of converting $\gamma$-rays by a Tungsten layer tracked by silicon microstrip detectors providing the two orthogonal coordinates for each element (point) along the track. AGILE-GRID event processing is operated by onboard trigger logic algorithms (Argan et al. 2004) and by on-ground event filtering. In this paper we use the on-ground GRID event filter called "F4" (Giuliani et al. 2006).

During its first year in orbit, AGILE surveyed the $\gamma$-ray sky and detected many galactic and extragalactic sources. The AGILE Commissioning phase ended on July 9, 2007, and the following science verification phase lasted about four months, up to November 30, 2007. On December 1, 2007 the baseline nominal observations and pointing plan of Cycle- 1 started with the guest observer program. In this paper we present the first catalog of high-confidence $\gamma$-ray sources detected by AGILE including data from July 9, 2007 to June 30, 2008, thus covering science verification phase data and the first seven months of the Cycle-1. Cataloged sources are detected by merging all the available data over the entire time period.

This is a significance-limited catalog that includes only sources above $4 \sigma$ extracted from the sample of AGILE detections obtained with a conservative data analysis, as described in the following sections. The catalog sensitivity is nonuniform, reflecting the inhomogeneous first-year AGILE sky coverage. The first-year exposure (see Fig. 1) was focused mainly towards the Galactic plane, mostly in the Carina-Crux and in the Cygnus regions. The average effective AGILE-GRID exposure time across the sky, for the chosen F4 event filter, is $\left\langle T_{\exp }\right\rangle \simeq 0.8 \times 10^{6} \mathrm{~s}$, with peak values of $\sim 7 \times 10^{6} \mathrm{~s}$. For a given statistical significance, the limiting point-source flux varies with position, owing to the diffuse $\gamma$-ray emission that represents a nonuniform background over which the pointlike sources are seen. The Galactic diffuse continuum $\gamma$-ray emission dominates other components and has a wide distribution with most emission coming from the Galactic 
plane. We detect limiting fluxes of about $2 \times 10^{-7} \mathrm{ph} \mathrm{cm}^{-2} \mathrm{~s}^{-1}$ with $\sim 4 \sigma$ statistical significance at galactic latitudes $|b|>10^{\circ}$.

The outline of the paper is as follows. In Sect. 2 we briefly discuss the AGILE-GRID response characteristics and in Sect. 3 describe the AGILE diffuse $\gamma$-ray model used in the data analysis. In Sect. 4 we describe the satellite pointing strategy and data flow. We then present in Sect. 5 the data reduction and analysis method used to build the first AGILE catalog. Our results and the list of detected high-confidence $\gamma$-ray sources are shown in Sect. 6. Finally in Sect. 7 we discuss our results and make some concluding remarks.

\section{AGILE-GRID response characteristics}

The AGILE-GRID inflight calibration during the first year and a half of observations has been recently completed and details of the instrument response characteristics will be given in Tavani et al. (2009b). The results are consistent with prelaunch simulations and instrument tests (Tavani et al. 2009a).

The energy-dependent in-flight GRID instrument point spread function (PSF) has a full-width at half-maximum $(F W H M)$ of approximately $3.5^{\circ}$ at $100 \mathrm{MeV}$, and gradually improves at higher energies. AGILE PSF is better than that of EGRET by a factor of $\sim 2$ above $400 \mathrm{MeV}$. The GRID effective area, as determined by inflight calibrations, reaches $500 \mathrm{~cm}^{2}$ at several hundred $\mathrm{MeV}$, depending on the GRID event filter used. The conservative event filter F4, chosen for our analysis, applies tight event selection cuts to eliminate a higher fraction of possible particle background counts. This filter is optimized to select $\gamma$-ray events within the central field of view (FoV) zone $\left(\sim 40^{\circ}\right.$ radius) at the expense of the effective area. In the energy range 200-400 MeV and at $\sim 30^{\circ}$ off-axis, the average effective area for the F4 filter is $\left\langle A_{\mathrm{eff}}\right\rangle_{(F 4)} \sim 300 \mathrm{~cm}^{2}$. It can be parametrized as a function of the off-axis angle $\theta$, in the range $\in\left[0 \div \theta_{M}\right]$, as

$\left\langle A_{\mathrm{eff}}(\theta)\right\rangle_{(F 4)}=A_{0}\left[1-\left(\frac{\theta}{\theta_{M}}\right)^{\alpha_{1}}\right]^{\alpha_{2}}$

where $A_{0}=366 \mathrm{~cm}^{2}, \theta_{M}=64^{\circ}, \alpha_{1}=3$, and $\alpha_{2}=2$.

Both AGILE PSF and effective area are characterized by a very good off-axis performance and are calibrated well up to almost $60^{\circ}$, showing very smooth variations with the angle relative to the instrument axis.

\section{AGILE diffuse gamma-ray model}

In the data analysis we use the AGILE diffuse emission model (Giuliani et al. 2004, 2009a) for diffuse $\gamma$-ray background-count predictions. Diffuse $\gamma$-ray emission includes a combination of two components: (1) diffuse emission from the Galactic interstellar medium and (2) an approximately isotropic extragalactic component, as well as possible contributions from unresolved and faint point sources. Diffuse emission coming from the Galactic plane dominates other components and, as in the EGRET model (Hunter et al. 1997), it is assumed to be produced by the interaction of cosmic rays with the interstellar medium through three physical processes: proton-proton collision, Bremsstrahlung, and inverse Compton emission.

The AGILE diffuse emission model substantially improves the previous EGRET model by using state-of-the-art neutral hydrogen (HI) and CO updated maps to model the matter distribution in the Galaxy. It is based on a 3 -D grid with $0.25 \times 0.25$ square degrees binning in Galactic longitude and latitude, and a
$0.2 \mathrm{kpc}$ step in distance along the line of sight. For the distribution of neutral hydrogen, we used the Leiden-Argentine-Bonn (LAB) Survey of Galactic HI (Kalberla et al. 2005). The LAB survey improves previous results especially in terms of sensitivity (by an order of magnitude), velocity range, and resolution. To properly project the velocity-resolved radio data, we used the Galactic rotation curves parameterized by Clemens et al. (1985). The detailed and relatively high-resolution distribution of molecular hydrogen is obtained from the $\mathrm{CO}$ observations described in Dame et al. (2001). The CO is assumed to be a tracer of molecular hydrogen, through a known ratio between hydrogen density and $\mathrm{CO}$ radio emissivity. Cosmic rays can emit $\gamma$-rays through the inverse Compton mechanism due to their interaction with photons of the cosmological background and of the interstellar radiation field (ISRF). To account for this component, we used the analytical model proposed by Chi \& Wolfendale (1991). It describes the ISRF as the result of three main contributions: far infrared (due to dust emission), near infrared, and optical/UV (due to stellar emission). The distribution of cosmic rays (both protons and electrons) in the Galaxy was obtained using the GALPROP cosmic-ray model (Strong et al. 2004; Moskalenko et al. 2007).

\section{AGILE data flow and Cycle-1 observational program}

AGILE satellite raw Telemetry data are down-linked approximately every $100 \mathrm{~min}$ to the ASI Malindi ground station in Kenya and transmitted first to the Mission Control Center at Telespazio, Fucino, and subsequently to the AGILE Data Center (ADC) for data reduction, scientific processing, and archiving. The ADC is the scientific component of the AGILE ground segment and is part of the ASI Science Data Center (ASDC) located in Frascati (Italy). The ADC includes scientific personnel from both the ASDC and the AGILE Team. More details on the ADC organization and tasks will be given in Pittori et al. (2009).

The AGILE pointings are subject to strict constraints requiring that the fixed solar panels always be oriented within $3^{\circ}$ from the Sun direction. AGILE pointings are called Observation Blocks (OBs) and usually consist of predefined long exposures, typically lasting 10-30 days, drifting about 1 degree per day with respect to the initial boresight direction to match solar panels illumination constraints. The large GRID FoV $(\sim 2.5 \mathrm{sr})$ and the low altitude orbit imply that, for most pointing directions, the Earth (partially) occults the FoV, thus the observing efficiency and exposure for a given source varies depending on its coordinates. To eliminate the Earth-albedo $\gamma$-ray contamination originating from interactions of cosmic rays with the upper atmosphere, a limb-angle cut was applied for all the $\gamma$-ray events with reconstructed directions less than $80^{\circ}$ with respect to the satellite-Earth vector. With this event selection we do not expect systematic effects caused by albedo photon-background fluctuations.

A predefined AGILE baseline pointing plan, aimed at reaching specific scientific goals that maximize the scientific output of the mission, is made public in advance at the AGILE web pages at $\mathrm{ASDC}^{1}$ to allow for the organization of multi-wavelength campaigns. Part of the AGILE science program is open to guest observers on a competitive basis through Announcements of Opportunity. Guest observers can apply for data which will be collected within the pointing plan. In case of Target of Opportunity ( $\mathrm{ToO}$ ) observations, the baseline plan is interrupted

\footnotetext{
${ }^{1}$ http://agile.asdc.asi.it
} 


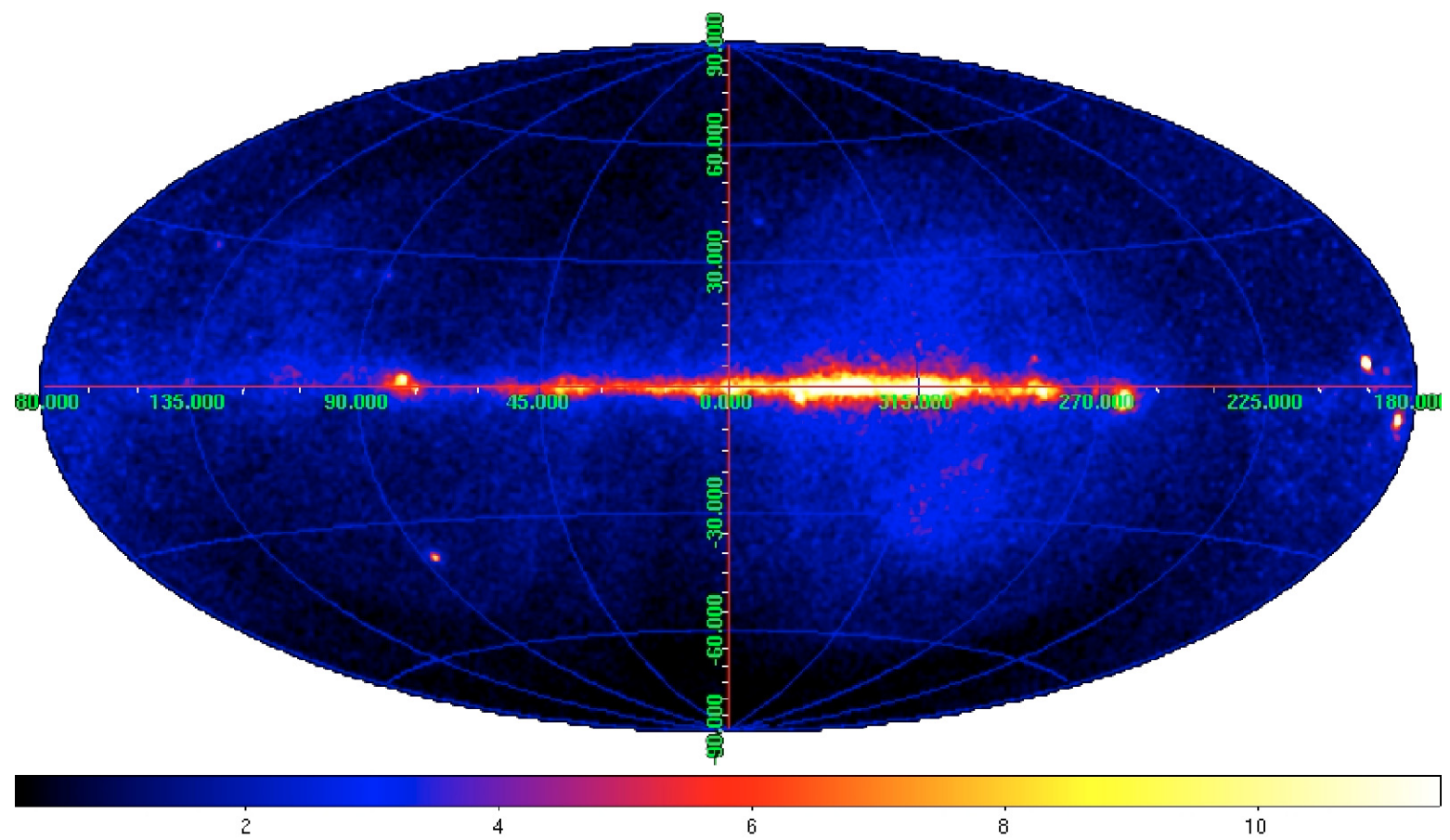

Fig. 2. Total AGILE-GRID count map in Aitoff projection and Galactic coordinates, for energies $>100 \mathrm{MeV}$ in units of $\mathrm{ph} \mathrm{cm}^{-2} \mathrm{~s}^{-1} \mathrm{sr}^{-1}$ accumulated during the period July 9, 2007-June 30, 2008 (with the F4 event filter). The effect of the nonuniform exposure is particularly evident for pointings centered near the Carina-Crux region.

and resumed at the end of the ToO, so that usually a ToO replaces some of the foreseen baseline pointings, and does not shift in time the execution of the remaining planned observations. The ADC web pages provide interactive tables for both the predefined AGILE baseline pointing plan and the actual list of pointings, including previously unforeseen ToOs.

In this paper we analyzed AGILE-GRID data of the 63 Observation Blocks reported in Table 2, covering the period from July 9, 2007 to June 30, 2008. The total $\gamma$-ray exposure and counts maps obtained over the selected period with the F4 filter, in Aitoff projection and Galactic coordinates, are shown in Figs. 1 and 2, respectively.

\section{AGILE data reduction and analysis}

Raw AGILE telemetry received at ADC is archived and transformed in FITS format through the AGILE Pre-Processing System (TMPPS) (Trifoglio et al. 2008). All GRID data are then routinely processed using the scientific data reduction software tasks developed by the AGILE instrument team and integrated into an automatic pipeline system developed at the ASI Science Data Center. The first step of the pipeline converts the satellite data time into terrestrial time (TT) on a contact-by-contact basis, and performs some preliminary calculations and unit conversions. A second step consists of the $\gamma$-ray event selection. We use an AGILE-GRID specific implementation of the Kalman Filter technique for track identification, event direction, and energy reconstruction (Giuliani et al. 2006; Pittori \& Tavani 2002). A quality flag is assigned to each event depending on whether it is recognized as a $\gamma$-ray event, a charged particle, a "singletrack" event, or an event of uncertain classification. An AGILE auxiliary (LOG) file is created, containing all the spacecraft information relevant to the computation of the effective exposure and livetime. Finally, the event directions in sky coordinates are reconstructed and reported in the AGILE event files (EVT), excluding events flagged as charged background particles. This step produces the Level-2 archive of LOG and EVT files on temporal intervals of few hours. A third step in the pipeline creates quick look (QL) counts, exposure, and diffuse $\gamma$-ray emission maps on different timescales: days, weeks, and daily increments on the OB timescale.

At the completion of each OB, we run the AGILE standard analysis $\mathrm{OB}$ pipeline that removes the data corresponding to repointing slews and occasional losses of fine-pointing attitude. GRID data used in our analysis have been processed with the standard software and in-flight calibrations available at the time of writing ${ }^{2}$. We used the high-quality F4 event filter ${ }^{3}$, whose response characteristics were described in Sect. 2.

The standardized and cleaned OB Level-2 archive is the basis for creating guest observers data packets and for the data merging used to build this first catalog.

\subsection{Data merging from the $O B$ archive}

To merge the data from different observing periods over the whole sky, we produced sets of FITS images in the ARC projection (Calabretta \& Greisen 2002) in Galactic coordinates, with a radius of $40^{\circ}$ and a bin size of $0.25^{\circ} \times 0.25^{\circ}$, oriented with the north Galactic pole facing upward. The centers of the maps were chosen according to the HEALPix (Hierarchical Equal Area isoLatitude Pixelization) algorithm (Górski et al. 2005) with $N_{\text {side }}=4$, for the coverage of the full sky with 192 maps, whose centers are at a constant latitude. The HEALPix algorithm produces a subdivision of a spherical surface in which each pixel covers the same surface area as every other pixel. However, the

${ }^{2}$ Software build version: BUILD GRID_STD_16 and BUILD GRID_SCI_15.2.

3 New filter algorithms highly efficient and optimized over a wider FoV have been developed and will be distributed by ADC during Cycle-2. 
HEALPix projection in FITS (Calabretta \& Roukema 2007) is not used here. Only the property of the HEALPix grid that the pixel centers occur on a discrete number of rings of constant latitude is used to represent all-sky $\gamma$-ray data binned in sky coordinates. The circular sky areas defined by a centroid and a radius constructed around the 192 HEALPix points are hereafter called "rings".

For each 12-h period, we produced maps of counts and exposure in the full energy band $E=100 \mathrm{MeV}-50 \mathrm{GeV}$ in rings yielding at least $20 \mathrm{~min}$ of effective exposure time within $30^{\circ}$ from each HEALPix point ${ }^{4}$. The 12 -h maps covering the whole sky were then summed over the entire one-year data span and analyzed with two independent source detection algorithms as described in the next section.

\subsection{Source detection method}

The AGILE source detection method is based on a maximum likelihood (ML) analysis to derive the best parameter estimates of source significance, $\gamma$-ray flux, and source location for each candidate source (Chen et al. 2009). The ML statistical technique, already used in the past in the analysis of $\gamma$-ray data (Mattox et al. 1996), compares measured counts in each pixel with the predicted counts derived from the diffuse $\gamma$-ray model to find statistically significant excesses consistent with the instrument point spread function. In the analysis we use the AGILE diffuse $\gamma$-ray model described in Sect. 3 for diffuse background (gas) map generation.

The likelihood ratio test is then used to compare the null (diffuse background-only) hypothesis with the possible presence of point-source components. According to Wilks' theorem (Wilks 1938), the point source "test statistic" (TS), defined as

$T S=-2\left(\ln L_{0}-\ln L_{1}\right)$

is expected to behave as $\chi_{1}^{2}$ in the null hypothesis, plus terms of order $O\left(N^{-1 / 2}\right)$, where $N$ is the number of counts. In practice for a number of AGILE counts $N>20$, the significance of a source detection at a certain position is given by a number of standard deviations $\sigma$ equal to $\sqrt{T S}$.

Our method for source detection consists of three steps:

1) preliminary automatic detection of counts map excesses and $\mathrm{ML}$ analysis on the resulting fixed positions. This step was performed with two independent detection strategies (A and $\mathrm{B}$ described below);

2) selection of high-confidence detections, according to the criteria described below;

3) refined analysis with a ML multi-source task to optimize source locations and flux estimates.

In step 1) two independent automatic source detection strategies over each ring count map were used:

A) identification of possible source locations using the standard Ximage $^{5}$ software for astronomical imaging (Giommi et al. 1992), adapted to $\gamma$-ray data analysis, and then single source

\footnotetext{
${ }^{4}$ We describe here our choice of parameters for map generation. To reduce the particle background contamination, only events tagged as confirmed $\gamma$-ray events were selected (filtercode $=5$ ). The South Atlantic Anomaly data were excluded (phasecode $=18$ ) and all the $\gamma$-ray events whose reconstructed directions with respect to the satellite-Earth vector is smaller than $80^{\circ}($ albrad $=80)$ were also rejected, to eliminate the Earth albedo contamination.

5 Ximage (Giommi et al. 1992) is part of the NASA's Heasarc Xanadu standard software package for multi-mission X-ray astronomy.
}

ML analysis (with the $A G \_$srctest_fixed task of the AGILE scientific pipeline). The Ximage detection algorithm locates point sources using a sliding-cell method so that positions and fluxes of each detected source are evaluated in a box maximizing the signal-to-noise ratio;

B) identification of possible source locations using a dedicated algorithm developed by the AGILE Team called SPOT based on a wavelet filtering technique adapted to $\gamma$-ray data (Bulgarelli et al. 2008), followed by a multi-source ML analysis (with the task $A G \_s r c l i s t$ ) used iteratively.

The AGILE SPOT algorithm used as method B) is a two-step procedure that extracts the excesses from counts maps and builds a list of candidate gamma-ray objects that are then analyzed by a likelihood method. To determine count excesses over the background, the SPOT algorithm analyzes the binned count maps with a smoothing of 1 degree, considers the bins with the largest number of counts, and adds to them the neighboring bins, thereby increasing the connected region, as described in (Di Stefano \& Bulgarelli 1999). The process ends when another connected region is merged with the first growing region. At that point, the merging step is reversed and two distinct connected regions are obtained. The centroids of all regions obtained in this way identify the positions of the gamma-ray candidate sources to be analyzed by a multi-source ML. Method A), which uses a single source likelihood analysis, typically optimizes detections of isolated $\gamma$-ray sources in extragalactic sky regions, whereas method $\mathrm{B}$ ) is more efficient in complex regions such as on the Galactic plane, where multiple source contributions may contaminate the result. In both cases we use an analysis radius of $10^{\circ}$ and a single power-law source model with spectral photon index $\alpha$. In our analysis we adopted a standard value of $\alpha=-2.1$, except for the Vela $\left(\alpha_{\text {Vela }}=-1.69\right)$ and Geminga $\left(\alpha_{\mathrm{Gem}}=-1.66\right)$ pulsars. This assumption is motivated by the known Crab-like spectral properties of the majority of EGRET sources and by the relatively small statistical significance of several AGILE sources, limiting our spectral analysis capability with chosen data sample. We postpone a detailed spectral analysis of the sources appearing in this catalog to forthcoming publications.

We populate two databases with all the results obtained with the automatic methods A) and B), and in step 2) we crosscorrelate the two subsamples extracted from the database with the following conditions:

- distance of the candidate source location from the center of the ring field of view has to be less than or equal to $30^{\circ}$, in order to perform the $10^{\circ}$ data analysis within the confidence region of the chosen F4 filter algorithm $\left(\leq 40^{\circ}\right)$.

- in each database we create a subsample by associating all the detections within a radius of $90^{\prime}$ to a single entry;

- for sources appearing in different ring areas, we select only detections with minimal distance from the center of the ring FoV;

- we select detections with $\sqrt{(T S)}>4$, which corresponds to a statistical significance of about $4 \sigma$.

We obtain

- 81 source candidates with source detection method A);

- 77 source candidates with source detection method B).

An initial cross-correlation radius of $90^{\prime}$ between the two dataset was used to select high-confidence galactic and extragalactic source candidates. 


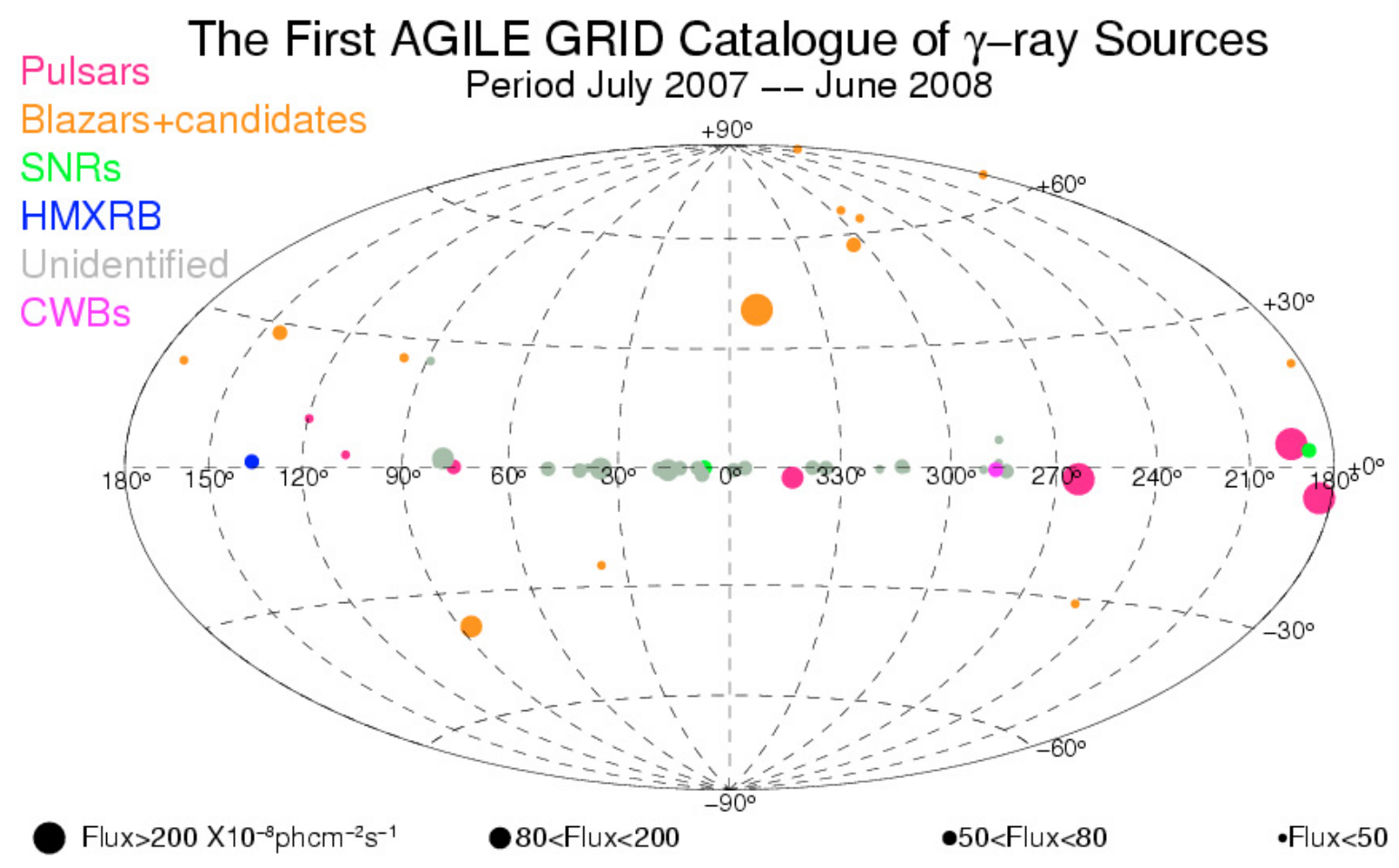

Fig. 3. The First AGILE-GRID Catalog of high-confidence sources, plotted in in Aitoff projection and galactic sky coordinates. Symbol sizes are proportional to source flux values, and symbol colors indicate different source classes.

Then in step 3), a manual refined analysis was performed with a multi-source likelihood analysis task, $A G \_m u l t i$, to confirm the detection and derive optimized source parameters. Special care should be used in particular on the galactic plane region $\left(|b|<10^{\circ}\right)$ to deal with possible source confusion and flux contamination. In the analysis of complex regions, positioning results obtained with detection methods A) and B) were also compared with a third standard peak detection software, SExtractor (Bertin \& Arnauts 1996), adapted to $\gamma$-ray data, using a wavelet filtering and deblending algorithm. We define "highconfidence" as those detections that pass all the requirements described in this section.

\section{First AGILE catalog of high-confidence gamma-ray sources}

The resulting list of validated sources, detected by using AGILEGRID data from July 9, 2007 to June 30, 2008 with the method and criteria described in Sect. 5, includes 47 high-confidence sources. The sources of this first AGILE catalog are plotted in Fig. 3 in galactic sky coordinates, classified in Table 1, including both confirmed and possible associations, and listed in Table 3.

In Table 1, for "confirmed" counterparts is meant $\gamma$-ray sources for which there are peer-reviewed publications demonstrating high-confidence association with refined analysis methods. Associations for uncertain sources were selected using cross-correlations with various updated public catalogs of $\gamma$, hard X-ray, and radio sources either specific mission or specific source classes, such as:

- the Third EGRET catalog (3EG) (Hartman et al. 1999) and the EGRET revised catalog of gamma-ray sources (Casandjian \& Grenier 2009);

- the INTEGRAL reference catalog (INTREFCAT) (Ebisawa et al. 2003);
Table 1. Census of the 47 First AGILE high-confidence gamma-ray sources.

\begin{tabular}{ccc}
\hline \hline Classification & Confirmed counterparts Possible counterparts \\
\hline Pulsar & 7 & 14 \\
Blazar FSRQ type & 4 & 3 \\
Blazar BL Lac type & 4 & - \\
Blazar Unknown type & - & 2 \\
CWB & 1 & - \\
SNR & 2 & - \\
HMXRB & 1 & 1 \\
Unidentified & - & 8 \\
\hline
\end{tabular}

- a selection from the Australian Telescope National Facility (ATNF) pulsar catalog (Manchester et al. 2003);

- HESS source catalog (available on-line);

- the SNR catalog (Green 1991; 2009);

- the Blazar Roma-BZCAT (Massaro et al. 2008).

Summing up, the first AGILE catalog includes 21 confirmed or candidate pulsars, 13 blazars (7 FSRQ, 4 BL Lacs, 2 unknown type), 2 HMXRBs, 2 SNRs, 1 colliding-wind binary system (CWB), and 8 unidentified sources.

In Table 3 we report the values of the following relevant source parameters:

- AGILE source name;

- source position both in celestial and galactic sky coordinates: RA, Dec (J2000), and LII, BII;

- position error $(95 \%)$, defined as the $2-\mathrm{D}$ error circle radius at $95 \%$ confidence level, statistical error only ${ }^{6}$;

- the $\sqrt{T S}$ values of the significance of the detection as determined from the refined ML analysis;

\footnotetext{
${ }^{6}$ The AGILE Team recommends adding a systematic error of $\pm 0.1^{\circ}$ linearly to this value.
} 
Table 2. AGILE pointings in the period 9 July 2007-30 June 2008, corresponding to the 63 Observation Blocks (OB) considered in our analysis. Acronyms used in table are: ToO = Target of Opportunity pointing, SA = SuperAGILE special pointing.

\begin{tabular}{|c|c|c|c|c|c|}
\hline Region name & OB number & Starting RA, Dec J2000 (deg) & Starting LII, BII (deg) & Start observation (UTC) & End observation(UTC) \\
\hline 3C 279 Region & OB900 & $195.596,-6.649$ & $307.8118,56.1183$ & 2007-07-09 12:00 & $2007-07-13 \quad 12: 00$ \\
\hline VELA Region & OB1000 & $157.979,-60.214$ & $286.4188,-1.8951$ & 2007-07-13 12:00 & 2007-07-24 12:00 \\
\hline ToO 3C 454.3 & OB1100 & $17.829,36.694$ & $127.3645,-26.0059$ & 2007-07-24 12:00 & 2007-07-30 12:00 \\
\hline ToO 3C 454.3 & OB1150 & $17.829,36.694$ & $127.3645,-26.0059$ & $2007-07-24$ 12:00 & 2007-07-30 12:00 \\
\hline VELA Region & OB1200 & $150.836,-70.19$ & $289.5293,-11.8265$ & 2007-07-30 12:00 & 2007-08-01 12:00 \\
\hline SA Crab -45 & OB1300 & $37.097,12.712$ & $156.5885,-43.7329$ & 2007-08-01 12:00 & 2007-08-02 12:00 \\
\hline VELA Region & OB1400 & $176.006,-66.063$ & $296.1593,-4.0824$ & 2007-08-02 12:00 & 2007-08-12 12:00 \\
\hline SA Crab -35 & OB 1500 & $47.41,16.075$ & $164.8343,-35.3162$ & 2007-08-12 12:00 & 2007-08-13 12:00 \\
\hline VELA Region & OB1600 & $195.551,-66.564$ & $304.0044,-3.7154$ & 2007-08-13 12:00 & 2007-08-22 12:00 \\
\hline SA Crab -25 & OB1700 & $57.139,18.566$ & $171.0790,-27.3115$ & 2007-08-22 12:00 & $2007-08-23$ 12:00 \\
\hline VELA Region & OB1800 & $216.979,-64.437$ & $313.1071,-3.4890$ & $2007-08-23$ 12:00 & 2007-08-27 12:00 \\
\hline Galactic Plane & OB1900 & $236.570,-41.874$ & $334.4369,10.0581$ & 2007-08-27 12:00 & 2007-09-01 12:00 \\
\hline SA Crab $(15,15)$ & OB2000 & $69.483,5.592$ & $190.8962,-26.2858$ & 2007-09-01 12:00 & 2007-09-02 12:00 \\
\hline $\operatorname{SACrab}(0,15)$ & OB2100 & $68.205,20.566$ & $177.1349,-18.2781$ & 2007-09-02 12:00 & 2007-09-03 12:00 \\
\hline SA Crab $(-15,15)$ & OB2200 & $66.651,35.559$ & $164.6334,-9.3529$ & 2007-09-03 12:00 & 2007-09-04 12:00 \\
\hline Field 8 & OB2300 & $51.408,71.022$ & $134.8816,11.8210$ & 2007-09-04 12:00 & 2007-09-12 12:00 \\
\hline SA Crab $(0,5)$ & OB2400 & $78.535,21.730$ & $182.1630,-9.8874$ & 2007-09-12 12:00 & 2007-09-13 12:00 \\
\hline Field 8 & OB2500 & $74.882,58.334$ & $150.9906,9.7255$ & $2007-09-13$ 12:00 & 2007-09-15 12:00 \\
\hline SA Crab $(45,0)$ & OB2600 & $84.212,-23.014$ & $226.7035,-26.1161$ & 2007-09-15 12:00 & 2007-09-16 12:00 \\
\hline $\operatorname{SACrab}(5,0)$ & OB2700 & $82.987,16.983$ & $188.5217,-8.9833$ & $2007-09-16$ 12:00 & 2007-09-17 12:00 \\
\hline SA Crab $(0,0)$ & OB2800 & $83.774,22.026$ & $184.6179,-5.6675$ & 2007-09-17 12:00 & 2007-09-18 12:00 \\
\hline SA Crab $(-5,0)$ & OB2900 & $84.62,27.048$ & $180.7737,-2.3343$ & 2007-09-18 12:00 & 2007-09-19 12:00 \\
\hline $\operatorname{SA~Crab~}(-15,0)$ & OB3000 & $85.347,37.089$ & $172.5873,3.5179$ & 2007-09-19 12:00 & 2007-09-20 12:00 \\
\hline SA Crab $(-25,0)$ & OB3100 & $86.174,47.118$ & $164.2603,9.2213$ & 2007-09-20 12:00 & $2007-09-21$ 12:00 \\
\hline SA Crab $(-35,0)$ & OB3200 & $87.140,57.126$ & $155.6110,14.6016$ & 2007-09-21 12:00 & 2007-09-22 12:00 \\
\hline $\operatorname{SA} C r a b(-45,0)$ & OB3300 & $88.348,67.136$ & $146.4473,19.4825$ & $2007-09-22 \quad 12: 00$ & $2007-09-2312: 00$ \\
\hline SA Crab $(0,-5)$ & OB3400 & $90.097,22.143$ & $187.5419,-0.5862$ & 2007-09-23 12:00 & 2007-09-24 12:00 \\
\hline SA Crab $(15,0)$ & OB3500 & $91.034,7.141$ & $201.1056,-7.1395$ & $2007-09-24$ 12:00 & $2007-09-25$ 12:00 \\
\hline SA Crab $(25,0)$ & OB3600 & $91.838,-2.882$ & $210.4602,-11.1195$ & $2007-09-25$ 12:00 & 2007-09-26 12:00 \\
\hline SA Crab $(35,0)$ & OB3700 & $92.502,-12.926$ & $220.0176,-14.9489$ & $2007-09-26$ 12:00 & 2007-09-27 12:00 \\
\hline Crab Nebula & OB3800 & $94.323,22.050$ & $189.5211,2.7938$ & 2007-09-27 12:00 & $2007-10-01$ 12:00 \\
\hline SA Crab $(0,-15)$ & OB3900 & $98.552,21.875$ & $191.4932,6.1922$ & 2007-10-01 12:00 & 2007-10-02 12:00 \\
\hline SA Crab $(-15,-15)$ & OB4000 & $100.839,36.784$ & $178.6417,14.3544$ & 2007-10-02 12:00 & $2007-10-03$ 12:00 \\
\hline SA Crab $(15,-15)$ & OB4100 & $99.566,6.788$ & 205.3927, 0.1791 & $2007-10-03$ 12:00 & 2007-10-04 12:00 \\
\hline Crab Field & OB4200 & $101.724,21.699$ & $192.9681,8.7550$ & 2007-10-04 12:00 & $2007-10-12$ 12:00 \\
\hline SA Crab $(0,-25)$ & OB4300 & $110.131,20.718$ & $197.2281,15.4667$ & 2007-10-12 12:00 & 2007-10-13 12:00 \\
\hline Gal. Center & OB4400 & $290.920,-18.896$ & $19.2683,-15.4110$ & $2007-10-13$ 12:00 & $2007-10-22$ 12:00 \\
\hline SA Crab $(0,-35)$ & OB4500 & $120.494,18.879$ & $203.0392,23.7444$ & $2007-10-22$ 12:00 & $2007-10-23$ 12:00 \\
\hline Gal. Center Reg. & OB4600 & $301.173,-17.107$ & $25.0972,-23.6663$ & $2007-10-23$ 12:00 & $2007-10-24$ 08:00 \\
\hline ToO $0716+714$ & OB4610 & $148.939,67.888$ & $143.3642,41.5875$ & 2007-10-24 08:00 & $2007-10-2912: 00$ \\
\hline ToO Extended & OB4630 & $157.461,66.942$ & $141.5537,44.7248$ & $2007-10-29$ 12:00 & 2007-11-01 12:00 \\
\hline SA Crab $(0,-45)$ & OB4700 & $130.614,16.339$ & $209.7914,31.7351$ & 2007-11-01 12:00 & 2007-11-02 12:00 \\
\hline Cygnus Region & OB4800 & $296.880,34.501$ & $69.5937,4.6227$ & 2007-11-02 12:00 & 2007-12-01 12:00 \\
\hline Cygnus Field 1 & OB4900 & $304.432,53.552$ & $88.8156,9.9272$ & 2007-12-01 12:00 & 2007-12-05 09:00 \\
\hline Cygnus Repointing & OB4910 & $322.496,38.244$ & $85.1187,-9.4171$ & 2007-12-05 09:00 & 2007-12-16 12:00 \\
\hline Cygnus Repointing & OB4920 & $322.496,38.244$ & $85.1187,-9.4171$ & 2007-12-05 09:00 & $2007-12-16$ 12:00 \\
\hline Virgo Field & OB5010 & $173.433,-0.437$ & $265.6464,56.7005$ & 2007-12-16 12:00 & 2008-01-08 12:00 \\
\hline Vela Field & OB5100 & $147.060,-62.517$ & $283.4703,-6.7881$ & 2008-01-08 12:00 & 2008-02-01 12:00 \\
\hline South Gal Pole & OB5200 & $58.347,-37.795$ & $240.3889,-50.5780$ & 2008-02-01 12:00 & 2008-02-09 09:00 \\
\hline ToO MKN 421 & OB5210 & $250.974,50.293$ & $77.3096,40.6278$ & 2008-02-09 09:00 & 2008-02-12 12:00 \\
\hline South Gal Pole Repointing & OB5220 & $65.660,-35.714$ & $237.5007,-44.6737$ & 2008-02-12 12:00 & 2008-02-14 12:00 \\
\hline Musca Field & OB5300 & $191.934,-71.893$ & $302.6408,-9.0241$ & 2008-02-14 12:00 & 2008-03-01 12:00 \\
\hline Gal. Center 1 & OB5400 & $243.596,-50.979$ & $332.1063,0.0207$ & 2008-03-01 12:00 & 2008-03-16 12:00 \\
\hline Gal. Center 2 & OB5450 & $265.781,-28.626$ & $359.9782,0.6280$ & 2008-03-16 12:00 & 2008-03-30 12:00 \\
\hline Anti-Center 1 & OB5500 & $100.944,21.711$ & $192.6369,8.1084$ & 2008-03-30 12:00 & 2008-04-05 12:00 \\
\hline SA Crab $(8,24)$ & OB5510 & $108.283,28.625$ & $188.9607,16.9953$ & 2008-04-05 12:00 & 2008-04-07 12:00 \\
\hline SA Crab $(15,26)$ & OB5520 & $111.762,35.688$ & $183.0072,22.2023$ & 2008-04-07 12:00 & 2008-04-08 12:00 \\
\hline Anti-Center 2 & OB5530 & $110.404,20.758$ & $197.2962,15.7167$ & 2008-04-08 12:00 & 2008-04-10 12:00 \\
\hline Vulpecula Field & OB5600 & $286.259,20.819$ & $53.0394,6.4733$ & 2008-04-10 12:00 & 2008-04-30 12:00 \\
\hline North Gal Pole & OB5700 & $250.075,72.497$ & $104.8522,35.4379$ & 2008-04-30 12:00 & 2008-05-10 12:00 \\
\hline Cygnus Field 2 & OB5800 & $304.286,35.974$ & 74.0497, 0.2720 & 2008-05-10 12:00 & 2008-06-09 18:00 \\
\hline ToO WComae ON+231 & OB5810 & $182.285,29.614$ & $195.5016,80.3738$ & 2008-06-09 18:00 & 2008-06-15 12:00 \\
\hline Cygnus Repointing & OB5820 & $323.248,50.079$ & $93.6645,-1.1664$ & 2008-06-15 12:00 & 2008-06-30 12:00 \\
\hline
\end{tabular}




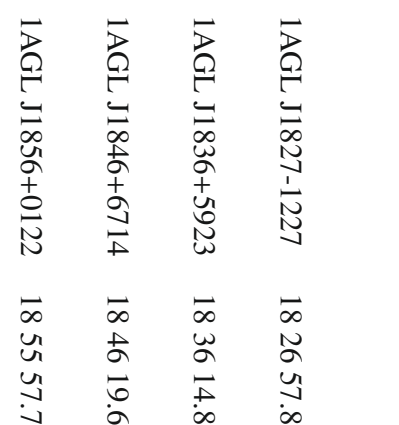

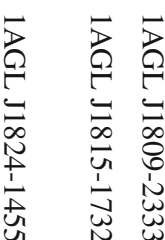

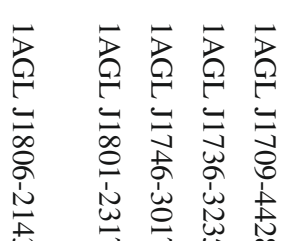

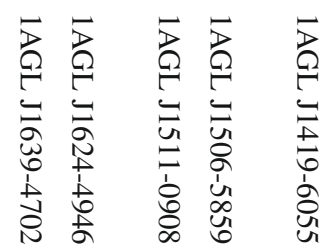

$\vec{\infty} \quad \vec{\infty}$

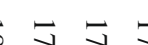

๙

$\vec{u} \bar{a} \quad \vec{a}$

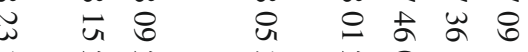

N

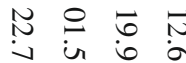

w

= 8

ir $\underset{i}{\infty} \stackrel{\text { in }}{\text { in }}$

$\pm \quad+\quad$ 苑

N $\vec{N}$ 滎

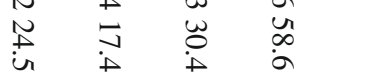

$\frac{1}{+} \stackrel{1}{\sim}$

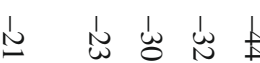

u w w

w $\exists \exists$ u

N.

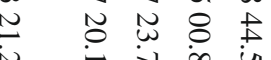

t

$\begin{array}{lll}1 & 1 \\ 0 & d & 0\end{array}$

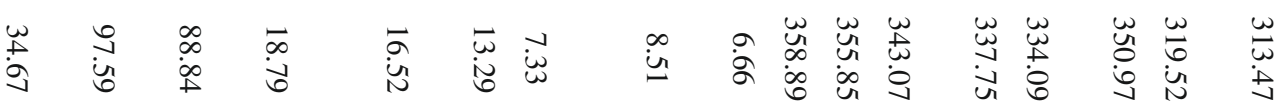

古 \&

i

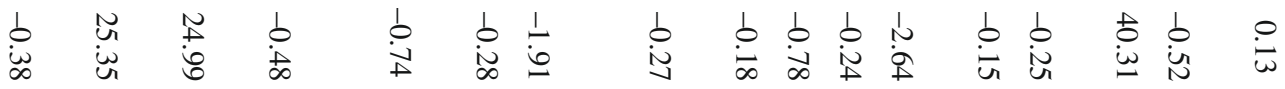

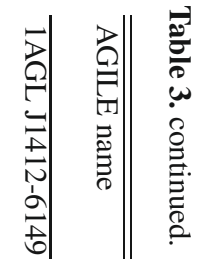

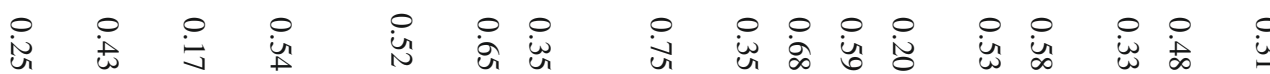

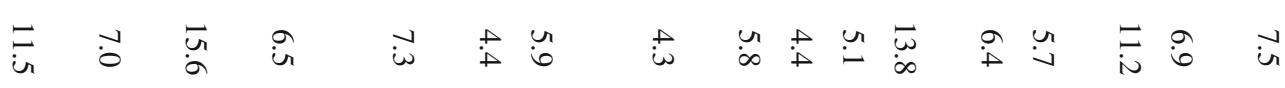

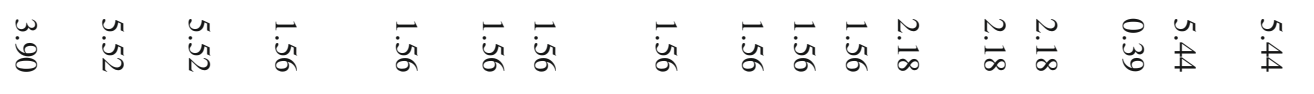

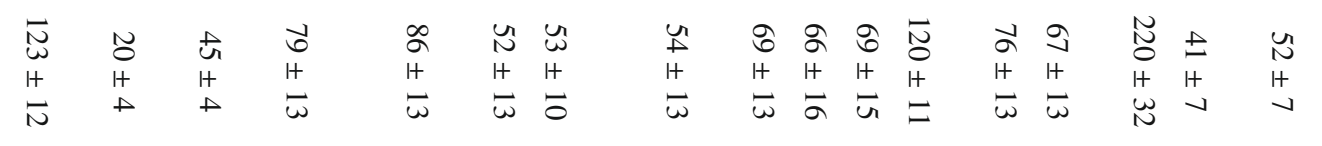

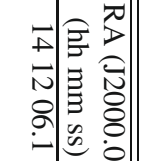
(1)

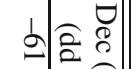

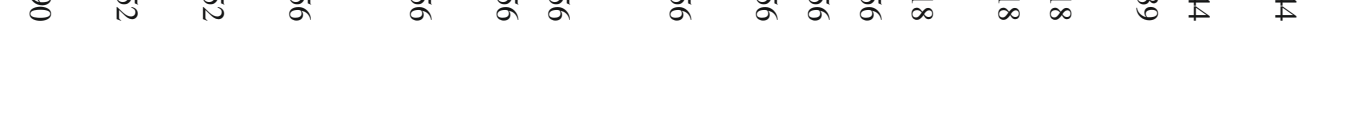

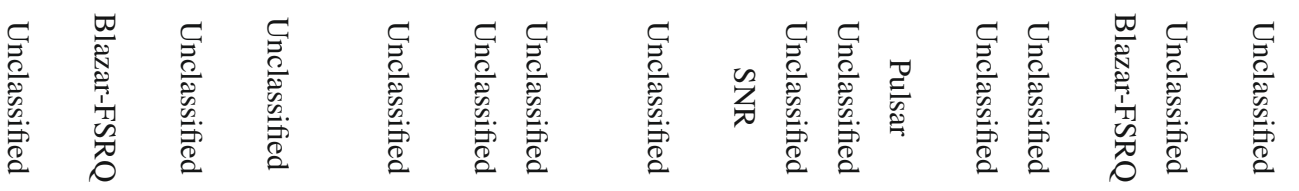

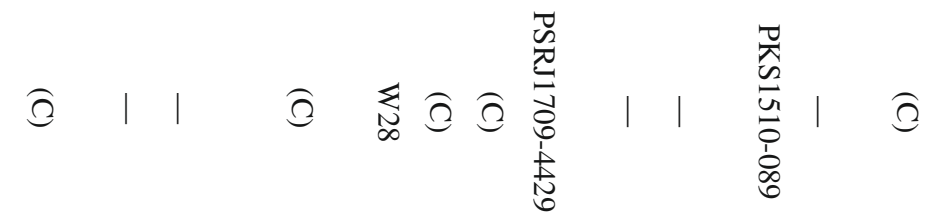

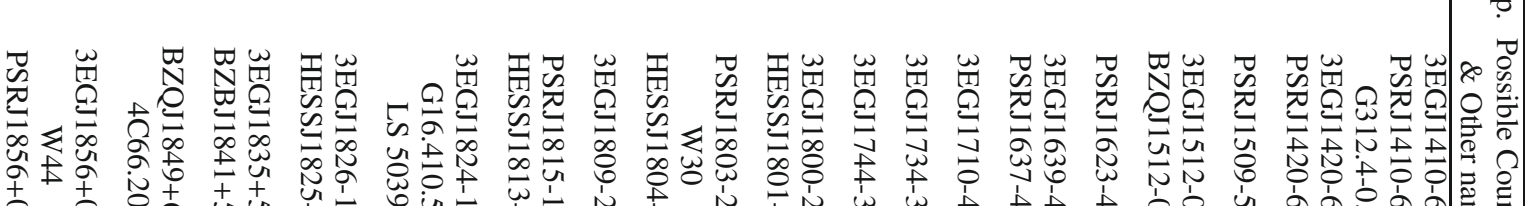




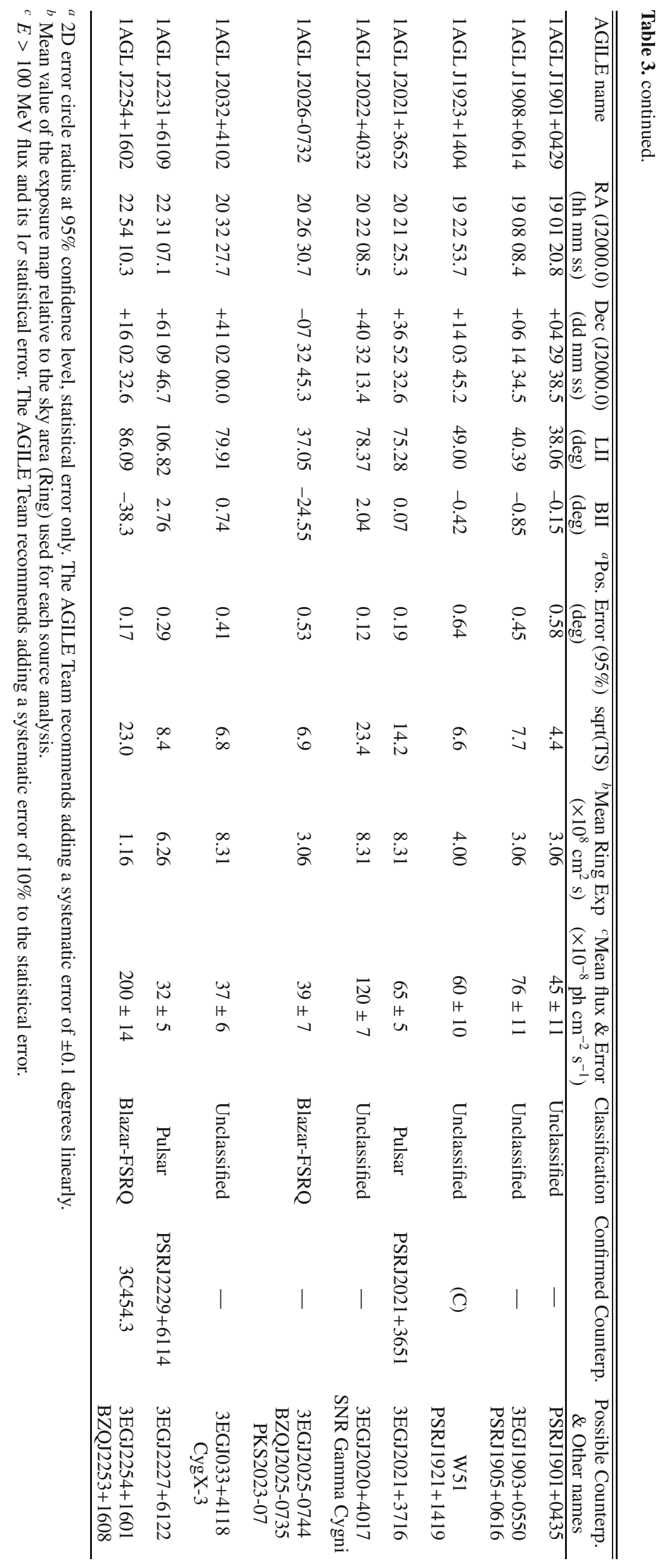


- the mean value of the F4 exposure map in units of $10^{8} \mathrm{~cm}^{2} \mathrm{~s}$, relative to the sky area (Ring) used for each source analysis;

- the source flux above $100 \mathrm{MeV}$ and its $1 \sigma$ statistical error ${ }^{7}$ in units of $10^{-8} \mathrm{ph} \mathrm{cm}^{-2} \mathrm{~s}^{-1}$. This is the average source flux value over the entire time period;

- source classification;

- counterpart name for confirmed sources;

- possible counterparts in the AGILE error radius and other names, both for "confirmed" and "uncertain" counterparts.

\subsection{Notes on individual sources}

As described in Sect. 5, pointlike $\gamma$-ray sources parameters reported in this paper are determined by a likelihood analysis of the $10^{\circ}$ field surrounding the candidate sources. The analysis depends on the local Galactic diffuse emission, the $\gamma$-ray photon statistics, the instrument PSF, the response matrix as a function of energy and off-axis angle, and on the background filtering. Particular care is required to carry out the analysis in regions of the Galactic plane that are characterized by a relatively high and structured flux of the diffuse Galactic emission, as well as in regions harboring nearby $\gamma$-ray sources leading to possible source confusion. For such regions we insert the label (C), for "Confused", in the Confirmed Counterpart column of Table 3. These are significant AGILE detections, which however have flux and location parameters that may be affected (within the statistical+systematic errors) by other nearby sources. In the following we briefly comment on some specific AGILE detections.

1AGL J0006+7311. This AGILE source, positionally coincident with the EGRET $\gamma$-ray source 3EG J0010+7309 located in supernova remnant CTA 1 , is associated with the first radio quiet pulsar recently discovered through its $\gamma$-ray pulsations by the Fermi Gamma-Ray Space Telescope (Abdo et al. 2008). This new class of young pulsar sources may be possibly associated with most unidentified Galactic $\gamma$-ray sources in star-forming regions and SNRs. Search for pulsations in $\gamma$-ray AGILE data is currently under way. At the border of the AGILE error box, there is also the blazar source BZQ J0019+7327.

1AGL J0535+2205 and 1AGL J0634+1748 (Crab and Geminga). These two well known strong $\gamma$-ray pulsars, together with the Vela pulsar, were used for in-flight AGILE calibrations. We report the flux values obtained during calibration subperiods. These values agree with pulsed flux values reported in (Pellizzoni et al. 2009). We note, however, that we observed higher flux values, over $1 \sigma$ from the reported mean flux, for both sources when merging all the data, including shorter ( 1 day) integration periods during 2007. This point is under investigation.

1AGL J0617+2236. This AGILE detection provides an improved positioning compared to the 3EG J0617+2238 error box. This source is positionally coincident with the SNR IC443 (Tavani et al. 2009c). The AGILE error box also contains the PSR J0614+2229.

1AGL J0657+4554 and 1AGL J0714+3340. These two highlatitude $(|b|>10 \mathrm{deg})$ AGILE sources, associated with blazars

7 The AGILE Team recommends adding a systematic error of $10 \%$ to the flux statistical error. of unknown type in the BZCAT, have no EGRET counterparts probably owing to flux variability.

1AGL J0835-4509 (Vela pulsar). As the most luminous steady source in the $\gamma$-ray sky, Vela has been extensively used for in-flight AGILE-GRID calibrations. With the F4 filter version and the strict criteria used to build this first catalog, the resulting effective exposure is quite low (only about $0.81 \times 10^{8} \mathrm{~cm}^{2} \mathrm{~s}$ on source over the entire period).

1AGL J1022-5822. This source lies in the complex Carina region, and multiple source contributions are possible.

1AGL J1043-5931. This source (not detected by EGRET) is close to 1AGL J1043-5749 in the Carina region. Our refined analysis leads to the association of this $\gamma$-ray source with the colliding wind binary Eta Carinae (Tavani et al. 2009d).

1AGL J1104+3754 and 1AGL J1222+2851. The effective exposure on these sources is low, just about 2 effective days, but it includes a ToO period on the source W Comae.

1AGL J1412-6149 and 1AGL J1419-6055. This source lies in the complex Crux region, and multiple source contributions are possible.

1AGL J1511-0908. The total effective exposure on this source is very low, just about 2 effective days, but it includes a ToO period on the associated source PKS 1510-089.

1AGL J1736-3235, 1AGL J1746-3017. These sources are in the complex region around $10^{\circ}$ from the Galactic center, and multiple source contributions are possible. We emphasize the relatively small exposure of the Galactic center region achieved until June 30, 2008, which does not allow a deeper analysis of the complex $\gamma$-ray emission from the center of our Galaxy.

1AGL J1801-2317. This source is spatially coincident with the TeV source HESS J1801-233. Remarkably, they both appear to be associated with the northeastern section of the SNR W28 shell (Giuliani et al. 2009b).

\section{Conclusions}

The AGILE Cycle-1 pointing plan covered the whole sky focusing mainly toward the Galactic plane. The AGILE first catalog includes only high-significance sources characterized by a prominent mean $\gamma$-ray flux above $100 \mathrm{MeV}$ when integrated over the total exposure period 2007 July-2008 June. With our one-year long integration, only sources with "steady" flux values above $\sim 20 \times 10^{-8} \mathrm{ph} \mathrm{cm}^{-2} \mathrm{~s}^{-1}$ are detected over $4 \sigma$. Source detections during flaring state and determination of peak fluxes are not included in this catalog. This should be taken into account when comparing with the results of the third EGRET catalog, which includes detections over $4 \sigma$ in each of the EGRET viewing periods during its effective 6-year lifetime. An analysis of $\gamma$-ray detection by the AGILE-GRID on short timescales (several weeks, 1-week, days) is beyond the scope of this catalog, so will be published elsewehere. The AGILE-GRID spatial resolution reached with long exposures is substantially better than that of EGRET, and the total exposure accumulated by AGILE in several sky regions, particularly near the Galactic plane, is comparable to the one obtained by EGRET in 6 years effective time. It is then interesting to compare the relatively high-flux 
sources detected by AGILE with the equivalent sources of the third EGRET catalog. Many bright $\gamma$-ray sources detected by EGRET are confirmed by AGILE, which provides comparable or better positioning. AGILE in the first catalog detected five sources that were not present in the 3EG catalog: 3 blazars and 2 candidate pulsars. As expected from statistical detection effects and source variability, some of the prominent (with flux range $40-100 \times 10^{-8} \mathrm{ph} \mathrm{cm}^{-2} \mathrm{~s}^{-1}$ ) $3 E G$ sources in the Galactic plane are not detected by AGILE with mean flux values at a significance level that is sufficient to be included in this first catalog.

It is also important to note that the AGILE-GRID exposure in the selected period has been accumulated mostly in the CarinaCrux and in the Cygnus regions, with relatively low exposure at the Galactic center. This explains the relatively small number of sources in the Galactic center region.

Finally, taking into account that the AGILE first catalog is not a complete flux-limited sample and is affected by selection effects due to the assumed fixed value $(-2.1)$ of the unknown source spectral indices, we observe that with a limiting flux of about $2 \times 10^{-7} \mathrm{ph} \mathrm{cm}^{-2} \mathrm{~s}^{-1}$, the number and rate of $\gamma$-ray Blazars observed by AGILE (13 Blazars: 7 FSRQs and 5 BL Lacs) is roughly consistent with expectations from the EGRET $\log N-\log S$ (Özel \& Thompson 1996; Mücke \& Pohl 2000). A detailed study over a complete AGILE AGN sample will be performed in the future.

A variability study of the sources of this first catalog on different timescales will appear in Verrecchia et al. (2009).

Acknowledgements. The AGILE Mission is funded by the Italian Space Agency (ASI) with scientific and programmatic participation by the Italian Institute of Astrophysics (INAF) and the Italian Institute of Nuclear Physics (INFN). We acknowledge funds from ASI grant I/089/06/2.

\section{References}

Abdo, A. A., Ackermann, M., Atwood, W. B., et al. 2008, Science, 322, 1218 Argan, A., et al. 2004, in Science Symposium Conference Record, 375, 371 Atwood, W. B., Abdo, A. A., Ackermann, M., et al. 2009, ApJ, 697, 1071 Barbiellini, G., Bordignon, G., Fedel, G., et al. 2001, AIP Conf. Proc., 587, 754 Bennet, K., Lichti, G. G., Bignami, G. F., et al. 1977, A\&A, 56, 469 Bertin, E., \& Arnauts, S. 1996, A\&AS, 117, 393

Bignami, G. F., Boella, G., Burger, J. J., et al. 1975, Space Sci. Instr., 1, 245 Bulgarelli, A., Trifoglio, M., Gianotti, F., et al. 2008, Proceedings of Astronomical Data Analysis Software and Systems XVIII, Quebec, Canada, ADASS, ASP Conf. Ser.

Calabretta, M. R., \& Greisen, E. W. 2002, A\&A, 395, 1077

Calabretta, M. R., \& Roukema, B. F. 2007, MNRAS, 381, 865

Casandjian, J. M., \& Grenier, I. A. 2008, A\&A, 489, 849
Chen, A., et al. 2009, in preparation

Chi, X., \& Wolfendale, A. W. 1991, J. Phys. G, 17, 987

Clemens, D. P. 1985, ApJ, Part 1, 295, 422

Dame, T. M., Hartmann, D., \& Thaddeus, P. 2001, ApJ, 547, 792

Di Stefano, L., \& Bulgarelli, A. 1999, Proceedings of the 10th International Conference on Image Analysis and Processing (ICIAP 1999), 322

Ebisawa, K., Bourban, G., Bodaghee, A., Mowlavi, N., \& Courvoisier, T. J.-L. 2003, A\&A, 411, L59

Feroci, M., Costa, E., Soffitta, P., et al. 2007, NIM A, 581, 728

Fichtel, C. E., et al. 1975, ApJ, 198, 163

Gianotti, F., \& Trifoglio, M. 2001, in Astronomical Data Analysis Software and Systems X, ed. F. R. Harnden, Jr., F. A. Primini, \& H. E. Payne, ASP Conf. Ser., 238, 245

Giommi, P., Angelini, L., Jacobs, P., \& Tagliaferri, G. 1992, Astronomical Data Analysis Software and Systems I, ASP Conf. Ser., 25

Giuliani, A., Chen, A., Mereghetti, S., et al. 2004, Mem. SAIt Suppl., 5, 135

Giuliani, A., Cocco, V., Mereghetti, S., Pittori, C., \& Tavani, M. 2006, NIM A, 568,692

Giuliani, A., et al. 2009a, in preparation

Giuliani, A., et al. 2009b, in preparation

Green, D. A. 1991, PASP, 103, 209

Green, D. A. 2009, Bull. Astron. Soc. India, 37, 45

Górski, K. M., Hivon, E., Banday, A. J., et al. 2005, ApJ, 622, 759

Hartman, R. C., Bertsch, D. L., Bloom, S. D., et al. 1999, ApJS, 123, 79H

Hunter, S. D., Bertsch, D. L., Catelli, J. R., et al. 1997, ApJ, 481, 205

Kalberla, P. M. W., Burton, W. B., Hartmann, D., et al. 2005, A\&A, 440, 775

Labanti, C., Marisaldi, M., Fuschino, F., et al. 2006, SPIE, 6266, 62663

Labanti, C., Marisaldi, M., Fuschino, F., et al. 2009, NIM A, 598, 470

Manchester, R. N., Hobbs, A. T., \& Hobbs, M. 2005, AJ, 129, 1993

Massaro, E., Giommi, P., Leto, L., et al. 2008 [arXiv: 0810.2206], on-line interactive table available at http://www.asdc.asi.it/bzcat

Mattox, J. R., Bertsch, D. L., Chiang, J., et al. 1996, ApJ, 461, 396

Michelson, P. F. 2008, APS Meeting Abstracts, TSSP, L2002

Moskalenko, I. V., Strong, A. W., Digel, S. W., \& Porter, T. A. 2007, AIP Conf. Proc., 921, 490

Mücke, A., \& Pohl, M. 2000, MNRAS, 312, 177

Özel, M. E., \& Thompson, D. J. 1996, AJ, 463, 105

Pellizzoni, A., Pilia, M., Possenti, A., et al. 2009, ApJ, 691, 1618

Perotti, F., Fiorini, M., Incorvaia, S., Mattaini, E., \& Sant'Ambrogio, E. 2006, NIM A, 556, 228

Pittori, C., \& Tavani, M. 2002, NIM A, 488, 295

Pittori, C., et al. 2009, in preparation

Prest, M., Barbiellini, G., Bordignon, G., et al. 2003, NIM A, 501, 280

Strong, A. W., Moskalenko, I. V., \& Reimer, O. 2004, ApJ, 613, 962

Tavani, M., Barbiellini, G., Argan, A., et al. 2008, for the AGILE Collaboration, NIM A, 588, 52 [arXiv: 0807.4254]

Tavani, M., Barbiellini, G., Argan, A., et al. 2009a, for the AGILE Collaboration, A\&A, 502, 1015

Tavani, M., et al. 2009b, in preparation

Tavani, M., et al. 2009c, in preparation

Tavani, M., Sabatini, S., Pian, E., Bulgarelli, A., et al. 2009d, ApJ, 698, L142

Thompson, D. J., Bertsch, D. L., Fichtel, C. E., et al. 1993, ApJS, 86, 629

Trifoglio, M., Bulgarelli, A., Gianotti, F., et al. 2008, Proc. SPIE, 7011, 70113E Wilks, S. S. 1938, Ann. Math. Stat., 9, 60 